\title{
EVALUACIÓN ECONÓMICA Y FINANCIERA DE LA IMPLEMENTACIÓN DE UN BIODIGESTOR EN UN TAMBO DE LA CUENCA LECHERA SANTAFESINA
}

\author{
Rostagno, M. N. ${ }^{1}$; CAstignani, M. I. ${ }^{2}$ : Mansilla, M. ${ }^{3}$ \\ ROSSLER, N. ${ }^{2}$ \& OSAN, O. ${ }^{2}$
}

\begin{abstract}
RESUMEN
El objetivo del trabajo es evaluar la factibilidad técnica y conveniencia económica de la instalación de un biodigestor para la obtención de biogás y biofertilizante como alternativa para el tratamiento de efluentes en un tambo de la cuenca lechera central santafesina, considerado como un caso de estudio. Se valora la contaminación generada actualmente a partir de los efluentes, situación sin proyecto, y se la compara con el beneficio proyectado a partir de las inversiones propuestas derivado del ahorro en compra de fertilizantes y de la generación de biogás. El proyecto es técnica, económica y financieramente factible para el caso analizado. El uso de biodigestores es una interesante alternativa para dar valor a los residuos orgánicos.
\end{abstract}

Key words: efluentes, biodigestor, biogás, biofertilizante, conveniencia económica.

\begin{abstract}
Economic and financial evaluation of a biodigester implementation in a dairy farm in the dairy basin of Santa Fe.

The objective of this paper is to evaluate the technical feasibility and economic convenience of a biodigester installation, to obtain biogás and biofertilizer as an alternative for the treatment of effluents in a dairy farm in the central dairy basin of Santa Fe, considered as a case study. The pollution generated currently from the effluents is assessed, a situation without a project, and compared
\end{abstract}

1.- Ingeniero Agrónomo. Asesor privado. San Francisco, provincia de Córdoba. Tel: +54 (03564) 15594562. Email: rostagnomartin@gmail.com

2.- Grupo de Economía Agraria Facultad de Ciencias Agrarias (UNL). Kreder 2805. (3080HOF) Esperanza, provincia de Santa Fe. Argentina. Telefax +54 (3496) 426400. Int. 155.

3.- Alumno de la Maestría en Negocios Agroalimentarios (FCA-FCE UNL), Coordinador del programa Graduado Embajador, UNL. Bv. Pellegrini 2750 - (3000) Santa Fe. Argentina. Tel: +54 (0342) 4571110 Manuscrito recibido el 23 de octubre de 2019 y aceptado para su publicación el 2 de marzo de 2020.

Rostagno, M. N.; Castignani, M.I.; Mansilla, M.; Rossler, N. \& Osan, O. Evaluación económica y financiera de la implementación de un biodigestor en un tambo de la Cuenca Lechera Santafesina. FAVE - Ciencias Agrarias 19 (1): 67-79. CC BY-NC-SA 4.0 (c)(1)(2) 


\section{N. Rostagno et al.}

with the projected benefit from the proposed investments derived from the savings in the purchase of fertilizers and the generation of biogás. The project is technically, economically and financially feasible for the case analysed. The use of biodigesters is an interesting alternative to give value to organic waste.

Key words: effluents, biodigester, biogás, biofertilizer, economic convenience.

\section{INTRODUCCIÓN}

La intensificación de los sistemas de producción ganaderos ocurrida en los últimos años, acrecentó la necesidad de desarrollar herramientas para el manejo de los residuos que permitan minimizar los impactos negativos sobre el ambiente, dar respuesta a las crecientes demandas de la población y de los mercados y cumplir con las reglamentaciones vigentes.

Durante el proceso productivo de la actividad lechera, se generan subproductos o residuos (efluentes, gases de efecto invernadero -GEI ${ }^{1}$-, etc.) así como también olores e insectos, que son potencialmente contaminantes del ambiente (aire, suelo, agua).

Un informe realizado por la FAO (2017) expone que las contribuciones de la ganadería a las emisiones de GEI en el mundo ascienden a 7,1 mil millones de toneladas de dióxido de carbono $\left(\mathrm{CO}^{2}\right)$ equivalente por año, siendo la ganadería responsable del $14,5 \%$ de las mismas. Más específicamente, la intensidad global de las emisiones del ganado lechero es de 2.128 millones de toneladas de $\mathrm{CO}^{2}$ equivalente. Por su parte, el Panel Intergubernamental de Cambio Climático (18), informa que la contribución de la ganadería bovina al calentamiento global es del orden del $10 \%$ y solo la mitad es atribuible a las vacas. El aumento de las concentraciones de los GEI en la atmósfera, por encima de los niveles naturales, incrementa el efecto invernadero generando consecuencias negativas sobre el clima. De acuerdo a Cardona-Iglesias et al (2017), la síntesis y emisión de metano en bovinos está vinculada al sistema de producción, al tipo de dieta ofrecido y la eficiencia productiva de los animales. Por lo tanto, las medidas que permiten reducir estas emisiones, están relacionadas al uso de alimentos de calidad, la salud animal, la gestión del estiércol de los rodeos y el uso energético eficiente (16).

Por otro lado, el aumento del tamaño de los rodeos y del confinamiento, hizo que las excretas que antes se distribuían en los lotes, ahora se concentren en mayor proporción en las instalaciones de ordeño y corrales de encierro, lo que requiere de nuevos sistemas para su manejo. Las alternativas para el tratamiento y gestión de efluentes son variadas, desde el almacenamiento del efluente en lagunas hasta la transformación total de residuos en productos útiles para el productor como biogás, energía, abono, reutilización del agua (2). Actualmente, algunos tambos incluyen algún tratamiento en

1.- GEI. Gases de efecto invernadero: vapor de agua, dióxido de carbono, metano, óxido de nitrógeno y ozono. 
el manejo de estos efluentes. No obstante, el aprovechamiento de los mismos es un poco más frecuente, pero de ninguna manera generalizado (5). La mayoría de los establecimientos lecheros de la región acumula los efluentes en lagunas que funcionan como depósitos y se vacían periódicamente esparciendo los líquidos en los lotes. Son escasos los tambos que han incorporado un sistema de gestión integral de sus efluentes, y se dispone de poca información sobre las particularidades y los resultados de los mismos. En el diseño y elección del sistema, es importante considerar la cantidad de agua utilizada y la cantidad y calidad del estiércol producido y posible de recolectar en las instalaciones y en los corrales de alimentación. La composición del estiércol varía de acuerdo con el tipo y edad del animal, el tipo de alimentación que recibe, el uso o no de cama, al manejo de las heces desde el momento en que son excretadas hasta que puedan ser utilizadas y al clima (3).

Los biodigestores aportan una solución práctica y alternativa en el manejo de los residuos orgánicos transformándolos en biofertilizantes, biogás o bioenergía. Existen muchas experiencias positivas sobre el uso de biodigestores en tambos de gran escala, siendo una tecnología menos probada a escalas más reducidas (1, 7, 8, 22, 2). El mercado ofrece una gran diversidad de biodigestores en cuanto a diseño, materiales, precio, capacidad, eficiencias de producción de energía, facilidad de mantenimiento, etc., la cuestión pasa por encontrar el que mejor se adapte a cada situación no solo en términos técnicos sino también económicos, considerando que la capacidad de pago no es la misma que en empresas grandes (17).

En este trabajo se propone evaluar la factibilidad técnica y económica de la instalación de un biodigestor a fin de mitigar los impactos ambientales negativos causados por la producción y agregar valor a los residuos generados. La propuesta de inversión se hace para un tambo ubicado en el Departamento Las Colonias, Provincia de Santa Fe, Argentina.

\section{MATERIALES Y MÉTODOS}

Para el cumplimiento del objetivo planteado se aplicó la metodología de estudios de casos (25), analizándose un establecimiento tambero del centro de la provincia de Santa Fe. Con este método los datos se obtienen desde una variedad de fuentes, tanto cualitativas como cuantitativas; esto es, documentos, registros de archivos, entrevistas directas, observación directa, observación de los participantes e instalaciones u objetos físicos (10). Lo resultados del estudio de un caso pueden generalizarse a otros que representen condiciones teóricas similares (23).

El establecimiento tambero en estudio se encuentra ubicado en la cuenca lechera central santafesina, a dos kilómetros de la ciudad de Esperanza. Es fundamental para este caso un correcto tratamiento de efluentes dada su ubicación cercana al distrito urbano. Cuenta con 563 hectáreas, divididas en tres unidades productivas: 215 hectáreas atribuidas a la producción tambera (77 hectáreas propias y las restantes arrendadas), 62 hectáreas a la recría (arrendadas) y 286 hectáreas a la agricultura (arrendadas), estas últimas en otra zona. Se trata de un sistema de base pastoril, con 350 vacas en ordeño y 50 vacas secas (Holando-Argentino) y una producción diaria de leche de aproximadamente de 8.500 litros. La familia empresaria manifiesta en sus objetivos producir de manera sustentable. 
Actualmente, los efluentes del tambo son derivados a una fosa de almacenamiento de 2 metros de profundidad que, al estar impermeabilizada, impide la contaminación de las aguas subterráneas y la lixiviación de nutrientes como el nitrógeno. Una bomba sumergible envía el contenido de la fosa a un carro estercolero que tiene una capacidad de 10.000 litros para, finalmente, esparcirlos en diferentes potreros del establecimiento con el objetivo de aprovechar los nutrientes, pero sin realizar un tratamiento previo. Esta acción tiene dos implicancias: por un lado, ciertos patógenos presentes en los efluentes pueden generar perjuicios sanitarios a los animales del predio y la posible transmisión potencial a humanos y, por otro lado, se subutilizan los recursos porque está perdiendo la posibilidad de obtener como producto de los desechos una importante cantidad de energía renovable, es decir, de gas metano, obteniendo un ahorro por la sustitución del gas envasado que actualmente debe comprar.

Para evaluar la factibilidad técnica, económica y financiera del proyecto privado se planteó una modelización a 5 años utilizando el software "Modelo para formular y evaluar proyectos ambientales en empresas lecheras”, desarrollado por los autores de este trabajo, cuya comunicación se encuentra en prensa (6). El mismo, contempla la valoración de la externalidad ambiental negativa generada por los efluentes sin tratar y permite evaluar la conveniencia de las inversiones contempladas en un plan ambiental a partir de la comparación de los saldos proyectados (ingresos vs egresos) de la situación sin proyecto, con los saldos de la situación con proyecto (en la que se incluyen las inversiones requeridas para la implementación del plan y los ingresos y egresos derivados de esas inversiones).
El modelo utiliza la metodología ABC (5), donde el criterio utilizado para la evaluación del proyecto es el Valor Actual Neto (VAN), valor monetario, expresado al momento de inicio del proyecto que muestra el resultado de un flujo de ingresos luego de descontar la inversión y el flujo de egresos a la tasa de descuento utilizada.

$$
\mathrm{VAN}=(-\mathrm{inv})+\frac{\mathrm{ING}_{1}-\mathrm{EGR}_{1}}{(1+\mathrm{r})}+\cdots+\frac{\mathrm{ING}_{\mathrm{n}}-\mathrm{EGR}_{\mathrm{n}}+\mathrm{VR}}{(1+\mathrm{r})^{\mathrm{n}}}
$$

El criterio de aceptación del proyecto de inversión fue que el resultado del VAN sea un valor mayor a cero, lo que indica que los ingresos generados por el proyecto permiten pagar la inversión realizada, los gastos de todos los períodos y descontando además la tasa de descuento seleccionada para el análisis.

Como criterio complementario se utilizó la Tasa Interna de Retorno (TIR) que es la tasa de interés en la que el VAN es cero. La TIR se compara con el costo de oportunidad de la inversión. La distancia entre la TIR hallada y la tasa de descuento utilizada en el cálculo del VAN es una medida de estabilidad de la decisión analizada.

Para mejorar las condiciones de decisión, se estimaron también el periodo de repago y la máxima inversión que soporta el proyecto evaluado. El primer criterio indica el tiempo, en años, que se necesitan para pagar la inversión inicial. El segundo, cual es el monto máximo, en términos monetarios, que puede pagarse la inversión sin que el VAN se vuelva negativo.

Los datos técnicos necesarios para la modelización se obtuvieron de consultas a informantes calificados; Para valorar el abono orgánico generado por el sistema de tratamiento de efluentes propuesto, se analizó el contenido de nutrientes del digestato (nitrógeno, fósforo, azufre, calcio) y se valo- 
raron a costo de oportunidad a partir del precio de la urea (para nitrógeno) y del fosfato diamónico (para fósforo) consultados en la revista Márgenes Agropecuarios del mes de abril de 2019, y del yeso agrícola (calcio y azufre) mediante consulta a comercios de la zona en abril de 2019, todos en dólares. El precio del gas generado por el digestor se obtuvo a partir de la conversión energética a gas propano o de garrafa consultado en comercios locales. El valor de las inversiones propuestas también fue obtenido mediante consulta a comercios locales.

\section{RESULTADOS Y DISCUSIÓN}

Kvolek (2018) recomienda como primer paso en la gestión ambiental, identificar y evaluar los impactos positivos o negativos generados por las actividades realizadas sobre el ambiente, para definir las medidas correctivas que permitan minimizar o contrarrestar los efectos y desarrollar un plan que contemple inversiones y prácticas que garanticen el cumplimiento de los estándares medioambientales Por lo tanto se describió, en primer lugar, la situación sin proyecto de la empresa estudiada y, posteriormente, se propusieron y evaluaron soluciones a los problemas ambientales generados.

Situación sin proyecto: Valor de la contaminación actual generada por emisión de metano

Para la valoración de la situación actual se consideró el impacto causado por la emisión de metano. No se valoraron otros posibles daños ambientales como la lixiviación de nitratos y nitritos hacia la capa freática o la generación de moscas y olores.

Se establecieron $75 \mathrm{~kg}$ anuales de metano producidos por cada vaca, siguiendo a Hilbert (2008), aunque los valores de producción y rendimiento de gas de los estiércoles presentan grandes diferencias entre distintos autores debido a la cantidad de factores que intervienen (19, 20, 9, 17, 24, 14). Entonces, esta empresa, genera anualmente 30.000 kg de metano que son liberados a la atmósfera contribuyendo como un componente de los gases de efecto invernadero. Para darle un valor económico a la emisión de metano se lo transforma de acuerdo a su capacidad calórica, a un equivalente energético como el gas propano (o gas de garrafa). Por su poder calorífico, $45 \mathrm{~kg}$ de gas propano equivalen a $58,4 \mathrm{~m}^{3}$ de gas metano, siendo la densidad de éste $0,743 \mathrm{~kg} / \mathrm{m}^{3}$. Por lo tanto, como se observa en la tabla 1, el valor en términos monetarios de la contaminación generada es de U\$S 27.690 anuales. Si este monto se considerara como un rubro adicional dentro de los costos de producción, tendría un impacto negativo sobre la rentabilidad de la empresa. Sin embargo, no es común que en las decisiones se considere el valor de la externalidad.

\section{Inversión propuesta para el tratamiento de efluentes: biodigestor}

Para disminuir la liberación del metano al ambiente y aprovechar parte del mismo, se propuso realizar un tratamiento anaeróbico de los efluentes generados, mediante la implementación de un biodigestor de flujo horizontal para obtener como subproductos biogás y biofertilizante. La finalidad básica es lograr la reducción de los parámetros físicos, químicos y biológicos de las aguas servidas, después de un periodo de entre 20

2.- Enargas https://www.enargas.gob.ar/ 
y 30 días en los digestores (17) que permitan obtener al final un efluente, cuyas características y calidad aseguren una baja o nula contaminación, medida en valores de demanda biológica de oxígeno (DBO) y demanda química de oxígeno (DQO) y puedan ser dispuestos al medio ambiente sin peligro para los cuerpos de agua receptores.

Para el dimensionamiento del biodigestor, se realizó un relevamiento de la cantidad de materia fecal que se podría obtener diariamente por vaca en la explotación en estudio. Se tomó como parámetro de referencia, por el tipo de explotación tambera (base pastoril), que entre el corral de espera y la sala de ordeño cada vaca deposita tres kilos de materia fecal fresca. Según la bibliografía consultada (14), con un kilo de materia fecal fresca de una vaca lechera, se podrían producir alrededor de 40 litros de biogás, aunque esto depende del contenido de sólidos volátiles del residuo que se utiliza como sustrato. Por lo que el tamaño del biodigestor a implementar es de $60 \mathrm{~m}^{3}$ con sistema de recirculación. El biodigestor considerado, en este caso, es el modelo Br 250 (de Biomax Solution), de tipo tubular, que tiene una maya interna especial para soportar presión, está recubierto con pvc y tratado contra rayos UV. La unidad cuenta con sus respectivas mangas de carga y descargas, válvula de salida de biogás, válvula y cañería de entrada y salida de los efluentes ${ }^{3}$. A fin de asegurar un buen diseño de la alimentación del biodigestor, se consideró un diámetro de tuberías de al menos $90 \mathrm{~mm}$ y automatizar el sistema de agitación a fin de que no existan problemas físicos para el normal funcionamiento de la tecnología a implementar. Además, para contribuir a la eficiencia de la digestión anaeróbica del efluente, se propuso enterrar el biodigestor hasta la mitad, con un posicionamiento sur-norte, para evitar el impacto que pueden tener las bajas temperaturas en la producción de biogás (12).

El monto de la inversión incluye el acondicionamiento del terreno, la construcción de una precámara de digestión y una pileta de almacenamiento del digestato, la conexión de tuberías, la bomba de carga y la bomba de agitación, más la conexión eléctrica y la automatización del sistema, además de la compra del biodigestor propiamente dicho. El costo total de la inversión del biodigestor en funcionamiento es de U\$S 6.500.

Tabla 1. Cuantificación económica de la contaminación por metano

\begin{tabular}{|l|c|c|}
\hline Vacas en ordeñe + vacas secas & Cab & 400 \\
\hline Generación de metano & $\mathrm{kg} / \mathrm{vaca}$ año & 75 \\
\hline Generación de metano & $\mathrm{kg} / \mathrm{año}$ & 30.000 \\
\hline Equivalente Gas propano & $\mathrm{kg} / \mathrm{año}$ & 31.112 \\
\hline Precio Gas propano & $\mathrm{U} \$ \mathrm{~S} / \mathrm{kg}$ & 0,89 \\
\hline Valor de la contaminación por generación de metano & $\mathrm{U} \$ \mathrm{~S} / \mathrm{año}$ & 27.690 \\
\hline
\end{tabular}

Fuente: Elaboración propia

3.- Biomax Solution. Biodigestor BR 250. http://www.biodigestores.com.ar/br-250/ 
Valoración de los beneficios del tratamiento de efluentes a partir de la instalación del biodigestor:

\section{- Producción de biogás a partir de las vacas en ordeño del establecimiento en estudio}

A partir de $1 \mathrm{~kg}$ de materia fecal fresca de vaca lechera, se puede producir 40 litros de biogás, que es un gas combustible cuyo poder calorífico oscila entre las 5.000 y $5.500 \mathrm{kcal} / \mathrm{m} 3$ debido a las variaciones en el contenido de metano, producto de las distintas mezclas de residuos orgánicos utilizados en cada biodigestor (15). El biogás obtenido a partir del estiércol de vacas lecheras contiene entre $65 \%$ y $70 \%$ de metano (gas natural), por lo tanto, con $1 \mathrm{~kg}$ de materia fecal fresca se producen 28 litros de gas natural. Este biogás se puede utilizar para la producción de energía eléctrica: 1.000 litros de biogás, con 65\% de metano, equivalen energéticamente a $6,41 \mathrm{kWh}^{-1}$ (15). El biogás generado puede utilizarse en cualquier equipo comercial para uso de gas natural (17).

En la tabla 2 se muestra la producción de biogás del establecimiento a partir de la instalación del biodigestor. Según estudios realizados por Hilbert (2008), por cada vaca lechera produciendo en un sistema pastoril, entre el corral de espera y la sala de ordeño se pueden obtener $3 \mathrm{~kg}$ de estiércol fresco.

Considerando los $1.050 \mathrm{~kg}$ de estiércol fresco generados, y que por cada kilo se producen 40 litros de biogás y que 1 litro de biogás equivale a 0,65 litros de gas natural, el potencial de producción del establecimiento rondaría los 7.665 kg/año de gas natural. Debido a que en los establecimientos rurales de Argentina no se cuenta con el servicio de gas natural, es común adquirir el gas propano envasado, generalmente en tubos de $45 \mathrm{~kg}$ que equivalen a 58,4m3 de gas natural. Considerado el precio del kg de gas propano, si el productor optara por la implementación de un sistema de digestión anaeróbica a través de un biodigestor, obtendría un ahorro anual de U\$S 6.821.

El establecimiento consume mensualmente, en promedio, cinco tubos de $45 \mathrm{~kg}$ de gas propano. Por lo tanto, el consumo anual de gas propano del establecimiento es de $2.700 \mathrm{~kg}$, generando un gasto de U\$S 2.403 .

Con estos datos se puede deducir que, mediante el potencial de producción de biogás del establecimiento, no solo alcanzaría para cubrir la demanda actual, sino también que tendría un excedente de gas propano de $4.965 \mathrm{~kg}$ equivalentes a U\$S 4.418,85 anuales. Este excedente puede ser envasado en garrafas y comercializado o puede alimentar un generador de combustión interna

Tabla 2. Producción de biogás en el establecimiento.

\begin{tabular}{|l|c|c|}
\hline Vacas en ordeño & cab & 350 \\
\hline Materia fecal recolectada & Kg/VO-día & 3 \\
\hline Biogás producido & $\mathrm{m}^{3} /$ día & 42 \\
\hline Equivalente en Gas natural & $\mathrm{m}^{3} /$ día & 27,3 \\
\hline Equivalente en Gas propano & $\mathrm{kg} / \mathrm{año}$ & 7.665 \\
\hline Precio Gas propano & $\mathrm{U} \$ \mathrm{~S} / \mathrm{kg}$ & 0,89 \\
\hline Ahorro anual & $\mathrm{U} \$ \mathrm{~S}$ & 6.821 \\
\hline
\end{tabular}

Fuente: Elaboración propia 
para generar electricidad, aunque esta última opción no es recomendable por la poca cantidad de biogás producida.

Al respecto, Castillo, A (2011) evaluó el uso de biodigestores para la producción de biogás por parte de productores familiares de Uruguay (en su mayoría queseros artesanales), destacando entre otras ventajas la mejora de la economía familiar a través de la reducción de gastos por la compra de combustibles derivados del petróleo.

- Generación de biofertilizantes a partir de las vacas en ordeño del establecimiento en estudio

El proceso de digestión anaeróbica estabiliza la materia orgánica de forma controlada al transformarla con valores de $\mathrm{pH}$ neutros $(6$ - 7,5$)$ lo que reduce la cantidad de sólidos volátiles. El biofertilizante obtenido (barros digeridos) no tiene olor y no atrae a las moscas (síntoma de que el biodigestor está funcionando correctamente) $\mathrm{y}$, aunque muy diluido, contiene los nutrientes originales del estiércol, que en el proceso anaeróbico han pasado de su forma orgánica a su forma mineral. Los nutrientes en forma orgánica (del estiércol fresco) no pueden ser asimilados directamente por la planta y requieren de una degradación, mientras que los ya mineralizados (biofertilizantes) pueden ser asimilados directamente por las plantas, lo que es una ventaja del biofertilizante (García Paez, 2019). Otra ventaja frente al estiércol fresco, es que la DQO se reduce hasta valores cercanos al 80\% (menor contaminación ambiental).

Para conocer las características del efluente del establecimiento, se tomó una muestra representativa de la fosa en donde es almacenado luego del ordeño y se envió para su análisis al Laboratorio de Servicios Analíticos de Desechos Agropecuarios, ubicado en la Facultad de Ciencias Agrarias de la Universidad Nacional del Litoral. Los resultados del análisis se reflejan en la tabla 3.

Los resultados del análisis sirven para obtener el dato de la proporción de macronutrientes (N, P, Ca, Mg, K) que se podrían recuperar para ser utilizados como biofertilizantes. En la tabla 4 se detallan las equivalencias y la producción total por nutriente.

Para la valoración del abono orgánico (biofertilizante) se calculó el contenido de los nutrientes más importantes (N, P, Ca, K, $\mathrm{Mg}$ ) y se valoraron a costo de oportunidad utilizando el precio de la UREA, DAP (Fosfato diamónico), Yeso Agrícola, en función del contenido de los nutrientes mencionados anteriormente. El ahorro total anual por el reemplazo de los fertilizantes inor-

Tabla 3. Resultados análisis de efluentes

\begin{tabular}{|c|c|c|}
\hline Determinaciones químicas & Valor & Técnica analítica \\
\hline Materia seca $(\%)$ & 4,09 & Secado en estufa a $70{ }^{\circ} \mathrm{C}$ \\
\hline Nitrógeno total $\left(\mathrm{mg} \mathrm{L}^{-1}\right)$ & 522,7 & Método Kjeldahl \\
\hline Fósforo total $\left(\mathrm{mg} \mathrm{L}^{-1}\right)$ & 92,2 & Colorimetría. Método del ácido ascórbico \\
\hline Calcio total $\left(\mathrm{mg} \mathrm{L}^{-1}\right)$ & 622,8 & Absorción Atómica \\
\hline Magnesio total $\left(\mathrm{mg} \mathrm{L}^{-1}\right)$ & 203,3 & Absorción Atómica \\
\hline Potasio total $\left(\mathrm{mg} \mathrm{L}^{-1}\right)$ & 343,6 & Absorción Atómica \\
\hline
\end{tabular}

Fuente: Elaboración propia en base a resultados provistos por el Laboratorio de Servicios Analíticos de Desechos Agropecuarios. 
Tabla 4. Equivalencia con Fertilizantes inorgánicos.

\begin{tabular}{|c|c|c|}
\cline { 2 - 3 } \multicolumn{1}{c|}{} & $\begin{array}{c}\text { Contenido } \\
\text { (gr/litro de efluente) }\end{array}$ & $\begin{array}{c}\text { Cantidad de nutriente } \\
\text { generada }(\mathrm{kg})\end{array}$ \\
\hline Nitrógeno & 0,52 & 1.803 \\
\hline Fosforo & 0,09 & 353 \\
\hline Calcio & 0,62 & 2.384 \\
\hline Magnesio & 0,2 & 767 \\
\hline Potasio & 0,34 & 1.315 \\
\hline
\end{tabular}

Fuente: Elaboración propia

Tabla 5: Producción de biofertilizantes y ahorro estimado anual (en dólares).

\begin{tabular}{|l|c|}
\hline Equivalente UREA $(\mathrm{kg})$ & 3.919 \\
\hline Equivalente DAP $(\mathrm{kg})$ & 1.763 \\
\hline Equivalente YESO AGRÍCOLA (kg) & 10.836 \\
\hline Ahorro estimado en equivalente UREA & 1.685 \\
\hline Ahorro estimado en equivalente DAP & 970 \\
\hline Ahorro estimado en Yeso Agrícola & 975 \\
\hline
\end{tabular}

Fuente: Elaboración propia

gánicos con los biofertilizantes es de U\$S 3.630 (tabla 5). Al igual que lo mencionado por García Paez (2019), en esta valoración económica de nutrientes, no se considera la materia orgánica que contiene el efluente y de la cual carecen por completo los fertilizantes sintéticos, lo que sería una ventaja adicional del uso de los biofertilizantes.

\section{Evaluación económica y financiera de} la instalación del biodigestor

Para realizar la evaluación del proyecto se realizó una proyección a 5 años, cuyo flujo se presenta en la tabla 6 .

El establecimiento en estudio cuenta con un tanque y una bomba estercolera por lo que, en la inversión de la instalación del biodigestor, no se contempló inicialmente la adquisición de esta maquinaria. La inversión total se propuso al inicio del proyecto (momento 0), con un valor de U\$S 7.000, donde además de la compra e instalación del biodigestor se contemplaron algunas reparaciones en la estructura del establecimiento. Se estimó un gasto anual, durante la vida del proyecto, de U\$S 500 en concepto de mantenimiento y posibles reparaciones del biodigestor. Los ingresos y egresos se mantuvieron fijos a lo largo de los 5 años debido a que las instalaciones de ordeño actuales se encuentran en su capacidad máxima y no permitirían un aumento del número de vacas. Los ingresos estimados por ahorro en el total de fertilizantes (UREA, DAP y Yeso) corresponden a la suma de los valores presentados en la tabla 5 . 
M. N. Rostagno et al.

Tabla 6. Flujo situación con proyecto (números expresados en dólares).

\begin{tabular}{|l|c|c|c|c|c|c|}
\hline & Momento 0 & Año 1 & Año 2 & Año 3 & Año 4 & Año 5 \\
\hline INGRESOS & & & & & & \\
\hline $\begin{array}{l}\text { Por ahorro estimado en compra de } \\
\text { fertilizantes }\end{array}$ & & 3.630 & 3.630 & 3.630 & 3.630 & 3.630 \\
\hline $\begin{array}{l}\text { Por ahorro estimado en el consumo } \\
\text { de Gas propano }\end{array}$ & 6.821 & 6.821 & 6.821 & 6.821 & 6.821 \\
\hline $\begin{array}{l}\text { Por disminución de emisión de } \\
\text { metano* }\end{array}$ & 7.000 & 6.922 & 6.922 & 6.922 & 6.922 & 6.922 \\
\hline INVERSIÓN TOTAL & & & & & & \\
\hline EGRESOS & & 20.767 & 20.767 & 20.767 & 20.767 & 20.767 \\
\hline $\begin{array}{l}\text { Gastos de mantenimiento y } \\
\text { reparaciones }\end{array}$ & & & & & & 2.800 \\
\hline $\begin{array}{l}\text { Egreso por liberación no controlada } \\
\text { de metano }\end{array}$ & -7.000 & -3.894 & -3.894 & -3.894 & -3.894 & -1.094 \\
\hline Valor Residual Pasivo** & & & 500 & 500 \\
\hline SALDO & & & & & \\
\hline
\end{tabular}

* Se estima una disminución de la eliminación de gas metano de $25 \%$ sobre el total. ** VRP $=40 \%$ del valor de la inversión. Fuente. Elaboración propia.

Tabla 7. Evaluación Proyecto (números expresados en dólares).

\begin{tabular}{|l|c|c|c|c|c|c|}
\hline & Momento 0 & Año 1 & Año 2 & Año 3 & Año 4 & Año 5 \\
\hline Saldo situación sin proyecto & & -27.690 & -27.690 & -27.690 & -27.690 & -27.690 \\
\hline Saldo situación con proyecto & -7.000 & -3.894 & -3.894 & -3.894 & -3.894 & -1.094 \\
\hline Beneficio incremental & -7.000 & 23.796 & 23.796 & 23.796 & 23.796 & 26.596 \\
\hline
\end{tabular}

\begin{tabular}{|c|cc|}
\hline Tasa de descuento & 10 & $\%$ \\
\hline VAN & 84.944 & U\$S \\
\hline TIR & 340 & $\%$ \\
\hline Periodo de repago & 1 & Años \\
\hline Inversión máxima & 91.944 & U\$S \\
\hline
\end{tabular}

Fuente. Elaboración propia.

En la tabla 7 se muestra el flujo de beneficio incremental del proyecto puro, obtenido como la diferencia entre los beneficios de las situaciones con y sin proyecto.

Se observa que, con una tasa de descuento del $10 \%$ (que representa el beneficio total anual esperado por el empresario), el VAN es positivo y supera ampliamente el valor de la inversión de U\$S 7.000. Lo mismo puede decirse de la TIR con respecto a la tasa de descuento considerada, diferencia que permite inferir una gran estabilidad del proyecto. Para completar el análisis económico-financiero, la inversión inicial se recupera en el transcurso del primer año y la inversión máxima que soporta el proyecto (inversión que hace igual a 0 el VAN) es de U\$S 91.944. Es importante mencionar que el proyecto es factible aun sin considerar el VRP en los cálculos (VAN = U\$S 83.206; 
TIR $=340 \%$ ) y en el caso de tener que adquirir el tanque y la bomba estercolera $($ VAN = U\$S 69.686; TIR = 85\% para un nuevo valor de la inversión total de U\$S 27.300 que incluye la adquisición de la maquinaria). Todos los criterios permiten decir que el proyecto es factible y presenta una estabilidad importante.

Al implementarse el proyecto, además de generarse ingresos extras al establecimiento (por ahorros en la compra de gas y fertilizantes), se podría mejorar la eficiencia productiva del mismo porque se utilizaría una materia prima que de otra manera terminaría siendo un desecho del sistema, mejorando los rendimientos de los cultivos, la calidad del suelo y reduciendo el impacto ambiental generado. Al respecto, Hilbert (2008) menciona que el biogás será viable en aquellas situaciones donde sus insumos tengan un bajo costo de oportunidad, la eficiencia del sistema sea la "adecuada" y los productos generados tengan un alto costo de oportunidad.

En este trabajo, a pesar de evaluarse un proyecto privado, se incluye el valor de las externalidades negativas generadas por la liberación de metano a la atmósfera, abordándose de esta forma (aunque de manera limitada) la perspectiva social que si fue evaluada por otros autores como De Prada y otros (2013) quienes analizan la necesidad de incluir la dimensión económica desde la perspectiva social en los estudios de impacto ambiental.

\section{CONCLUSIONES}

El objetivo fue evaluar la conveniencia económica y financiera de las inversiones requeridas para implementar un plan de medidas correctivas que mejore o mitigue los impactos ambientales negativos causados por la producción en un establecimiento lechero ubicado en el centro de la provincia de Santa Fe, que fue evaluado como caso de estudio.

A partir de los resultados logrados se concluye que es factible técnica, económica y financieramente llevar adelante el proyecto. Los biodigestores son una oportunidad para cambiar el manejo de los residuos orgánicos, porque estos sistemas no sólo ‘tratan' los residuos, y ayudan al reciclaje de nutrientes por medio del uso del fertilizante producido, sino que además ofrecen un aprovechamiento energético por medio de la captura y uso del biogás generado. Esto diferencia a los biodigestores de otras tecnologías de energías renovables, que solo producen energía, y de otros procesos de tratamiento de residuos orgánicos, que únicamente producen fertilizante para el reciclaje de nutrientes. La utilización del biogás, en reemplazo de combustibles fósiles, trae la ventaja de poder autoabastecer ciertos consumos internos del predio, tanto para las labores de ordeño, lavado y calentamiento de agua, evitando un gasto que en ciertas ocasiones llega a ser sustancial.

El problema ambiental causado por los efluentes del tambo puede constituirse en una oportunidad de negocio considerando su aprovechamiento, en una amenaza considerando los efectos ambientales si no se realiza un tratamiento adecuado. El manejo actual de los residuos representa una 
debilidad para el sistema de producción de leche. Muy pocos productores realizan una correcta gestión y manejo de efluentes; la gran mayoría los deposita en lagunas sin impermeabilizar y, en general, hay desconocimiento de la cantidad y calidad del efluente generado.

Finalmente, los biodigestores, no dejan de ser una herramienta, por lo tanto, es necesario analizar el contexto para poder fortalecer su uso.

\section{BIBLIOGRAFÍA}

1.- AQUA LIMPIA ENGINEERING. 2015. Aprovechamiento de estiércol de ganado para la producción de energía. Disponible en: https://onedrive.live.com/?authkey=\%21 A O95C0\%2DaZs6Ds\%5 FA \& cid $=70$ BC5F696834B0AE \& i d=70BC5F696834B0AE\%21591614\&parI$\mathrm{d}=70$ BC5F696834B0AE\%21403473\&o=OneUp Acceso: 06/02/2020.

2.- ARAOS MILLAR, R. 2018. Recomendaciones para rentabilizar un proyecto de biodigestión en el sector lechero. El Mercurio. Campo. Disponible en: https://www. elmercurio.com/Campo/Noticias/Noticias/2018/08/06/Como-rentabilizar-un-proyecto-de-biodigestion-en-el-sector-lechero. aspx?disp=1 Acceso: 06/02/2020.

3.- BONILLA CÁRDENAS, J. A. y LEMUS FLORES, C. 2012. Emisión de metano entérico por rumiantes y su contribución al calentamiento global y al cambio climático. Revisión. Rev. Mex. Cienc. Pecu. 3(2): 215-246.

4.- CARDONA-IGLESIAS, J. L.; MAHECHA-LEDESMA, L. y ANGULO-ARIZALA, J. 2017. Arbustivas forrajeras y ácidos grasos: estrategias para disminuir la producción de metano entérico en bovinos.
Agronomía Mesoamericana, 28(1): 273-288. Disponible en: https://dx.doi.org/10.15517/ am.v28i1.21466 Acceso: 06/02/2020.

5.- CASTIGNANI, M.I.; N. ROSSLER; $O$. OSAN; M. SUERO; M. TRAVADELO; M. MAINA Y M. C. BRIZI. 2016. Evaluación del impacto de la tecnología y el cuidado del ambiente sobre los resultados físicos y económicos en sistemas lecheros, a través de un modelo de mediano plazo. En: VICIEN, C.; S. PENA DE LADAGA; G. PETRI (Compiladores). Modelización económica en el sector agropecuario VI. Ed. Orientación Grafica. Buenos Aires, Argentina. p. 305-326

6.- CASTIGNANI, M.I.; ROSSLER, N.; OSAN, O.E.; MANSILLA, M.E. y ROSTAGNO, M. 2019. Modelo para formular y evaluar proyectos ambientales en empresas lecheras (En prensa).

7.- CASTILLOS, A. 2011. Biogás. Construcción y funcionamiento de biodigestores plásticos de flujo continuo. Programa de pequeñas donaciones del FMAM Uruguay. PNUD Uruguay. Disponible en: file://C:/ Users/Usuario/Downloads/Biogás-construccion\%20y\%20funcionamiento\%20biodigestores.pdf Acceso: 07/02/2020.

8.- CLARÍN. CAMBIO CLIMÁTICO. 2015. Energía con estiércol de vaca. Disponible en: https://www.clarin.com/cambio-climatico/cambio_climatico-gases_de_efecto_invernadero-dioxido_de_carbono-temperatura-estiercol-vaca-metano_0_BkVnnZYwQl. html Acceso: 10/02/2020

9.- CHASE, L. 2007. Methane emissions from dairy cattle. En: MUHLBAUER, E, MOODY, L Y BURNS, R. (Eds). Proceedings of mitigating air emissions from animal feeding operations conference. College of Agriculture and Life Sciences, IA, USA. p. 106-109. 
10.- CHETTY, S. 1996. The case study method for research in small- and médium - sized firms. International small business journal.

11.- DE PRADA, J. D.; GIL, H.A.; PEREYRA, C. I. Y BECERRA, V. H. 2013. La inclusión de la dimensión económica en la Evaluación de Impacto Ambiental. RIA 39(3): 259-266. Disponible en: file://C:/Users/ Usuario/Downloads/Dialnet-LaInclusionDeLaDimensionEconomicaEnLaEvaluacionDeI-4696612.pdf Acceso: 06/02/2020.

12.- DURAZNO CORONEL, A.D. 2018. Valoración de estiércol bovino y porcino en la producción de biogás en un biodigestor de producción por etapas. Tesis. Carrera de Ingeniería Ambiental. Universidad Politécnica Salesiana. Sede Cuenca. Cuenca. Ecuador.

13.- FAO. 2017. El trabajo de la FAO sobre el cambio climático. Conferencia de las Naciones Unidas sobre el cambio climático 2017. Disponible en http://www.fao.org/3/ai8037s.pdf Acceso: 07/02/2020

14.- FAO. 2019. Guía teórico-práctica sobre el biogás y los biodigestores. Colección Documentos Técnicos $N^{\circ} 12$. Buenos Aires. 104 pp.

15.- GROPPELLI, E. y GIAMPAOLI, $O$. 2016. Biodigestores: una propuesta sustentable. Ediciones UNL. Santa Fe. Argentina.

16.- HERRERO, M.A. Y THIEL, I. 2002. Water contamination from rural production systems. En: DONOSO, M.C. (Ed.). Water interaction with Energy, Environment, Food and Agriculture. Encyclopedia of Life Support Systems. Eolss publishers Co. Ltd. Oxford. United Kingdom. p. 168- 202.

17.- HILBERT, J.A. 2008. Manual para la producción de biogás. Instituto de Ingeniería Rural. Buenos Aires. INTA Castelar.

18.- IPCC. Total annual anthropogenic GHG emissions by gases 1970-2010. Disponible en: https://ar5-syr.ipcc.ch/ipcc/sites/default/ files/AR5_SYR_Figure_SPM.2.png Acceso: 06/02/2020.
19.- JOUANY, J. 1994. Methods of manipulating the microbial metabolism in the rumen. Annales de zootechnie, INRA/EDP Sciences, 43 (1): 49-62.

20.- KINSMAN, R.; SAUER, F.; JACKSON, H. y WOLYNETZ, M. 1995. Methane and carbon dioxide emissions from dairy cows in full lactation monitored over a six month period. J. Dairy. Sci. 78:2760-2766.

21.- KVOLEK, C.M. 2018. Gestión ambiental pecuaria. Aspectos teóricos y prácticos en el manejo de efluentes y uso agronómico de estiércol y efluentes. Editorial ORIENTACIÓN. Ciudad Autónoma de Buenos Aires. Argentina. 152p.

22.- LA NACIÓN. 2017. Con una inversión de US\$ 6 millones, Adecoagro inauguró un biodigestor para producir energía renovable con el tambo. Disponible en: https://www.lanacion.com.ar/economia/campo/con-una-inversion-de-us-6-millones-adecoagro-inauguro-un-biodigestor-para-producir-energia-renovable-con-el-tambo-nid2086788 Acceso: 06/02/2020.

23.- MARTÍNEZ CARAZO, P.C. 2006. El método de estudio de caso: estrategia metodológica de la investigación científica Pensamiento \& Gestión. 20 (julio): 165-193 Universidad del Norte Barranquilla, Colombia. Disponible en: https://www.redalyc.org/ articulo.oa?id=64602005 Acceso: 10/2/2020

24.- MOATE, P. 2010. Reducing methane emissions from dairy cows. Gippsland. How Now Gippy Now Septiembre Edition

25.- YIN, R.K. 1994. Case Study Research Design and Methods, Applied Social Research Methods (Vol. 5, 2nd ed.), Newbury Park, CA, Sage. 